\title{
Editorial on Conflict Resolution and Group Decision Based on Papers from the 2018 International Conference on Group Decision and Negotiation (GDN 2018)
}

\author{
Keith W. Hipel, ${ }^{a, b, c}$ Haiyan $X^{d}$

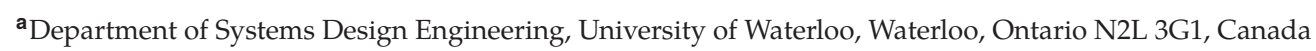 \\ ${ }^{b}$ Centre for International Governance Innovation, Waterloo, Ontario N2L 6C2, Canada \\ ${ }^{\mathrm{c} B a l s i l l i e}$ School of International Affairs, Waterloo, Ontario N2L 6C2, Canada \\ kwhipel@uwaterloo.ca (凶)

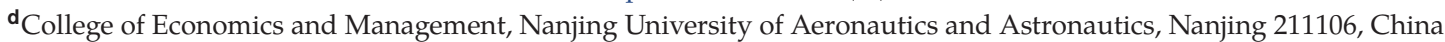 \\ xuhaiyan@nuaa.edu.cn
}

The key objective of this special issue of the Journal of Systems Science and Systems Engineering (JSSSE) is to assess papers containing leading-edge ideas on the theory and practice of conflict resolution and group decision making. Earlier versions of these fine papers were originally delivered by the authors at the 18th International Conference on Group Decision and Negotiation (GDN) which was held in Nanjing, China, from June 9 to 13, 2018 (GDN 2018), and which appeared as extended abstracts in a conference proceedings edited by Jiang et al. (2018) for which the authors of these abstracts maintain the copyrights. Subsequent to GDN 2018, authors were invited by the Guest Editors to prepare and submit significantly expanded versions of their papers for review and possible publication in this special issue of JSSSE. Following an overview of GDN 2018, the Guest Editors put the admirable contributions of the authors of these papers into perspective. In this way, readers will enjoy their academic journey through this landscape of fascinating concepts and challenging real-world applications as they learn about the many contributions of the authors in this important realm of conflict resolution and group decision.

The GDN Section comprises a dedicated body of scholars and practitioners, which is part of a highly respected international organization called INFORMS (The Institute for Operations Research and the Management Sciences). In response to the need to have a unique dedicated professional organization that represents members interested in addressing a challenging array of issues occurring in group decision and negotiation, Melvin Shakun founded the GDN Section and an associated journal within INFORMS in 1992. Starting in the year 2000 in the Scottish city of Glasgow, the GDN Section launched an annual series of conferences which has subsequently convened in cities around the globe on a yearly basis except for a one year gap when the "Arab Spring" blossomed in the Middle East. GDN 2018 constitutes the first occasion on which the GDN Section convened a conference in China or anywhere within Asia. In fact, under the fine leadership of Prof. Haiyan $\mathrm{Xu}$ and in concert with international and Chinese colleagues, GDN 2018 marks the largest GDN conference ever held thus far and, like its predecessors, was highly successful. The attractive venue in which GDN 2018 was held from June 9 to 13, 2018 was the Marriott Nanjing South Hotel located in the beautiful historical city of Nanjing. The Nanjing University of Aeronautics and As- 
tronautics (NUAA) was the generous sponsor of GDN 2018 with most of the local organizers being based in the College of Economics and Management (CEM) at NUAA.

The organizational team and Guest Editors are glad to report that more than 250 scholars participated in GDN 2018. Prior to the conference, over 140 submissions of extended abstracts and papers were received for review and possible presentation at GDN 2018. For release at the commencement of the conference on June 9, 2018, a local volume containing 108 abstracts organized into eight main topics was prepared (Jiang et al. 2018). Additionally, a Springer book entitled Group Decision and Negotiation in an Uncertain World having a total of 15 refereed full conferences papers was released (Chen et al. 2018). As just mentioned, this special JSSSE issue of papers first presented at GDN 2018 was put together by the Guest Editors after the conference was held. There are seven papers from GDN 2018 appearing in this special issue, which can be subdivided into two main categories: three papers falling under the classification of conflict resolution and four articles dealing with important topics in group decision making.

Consider first the three papers falling under the theme of conflict resolution, all of which deal with the Graph Model for Conflict Resolution (GMCR). In the first paper, Wu et al. (2019) design a mediation strategy for employment by a third party to bring two disputants together to achieve a reasonable resolution. In particular, within the structure of the matrix interpretation of the GMCR, the authors use inverse GMCR to ascertain the minimum preference change required to reach a reasonable result. Their procedure is applied to a conflict between a patient and a medical service provider.

Silva et al. (2019) are motivated by a local development conflict in Recife, Brazil, to design a procedure for obtaining the preferences of a DM, such as a regulatory body which is supposed to be unbiased. To achieve this, the authors design a procedure for integrating Dempster-Shafer Theory for combining expert knowledge and the Analytic Hierarchy Process (AHP) to rank the states for the regulator or DM being considered. They employ their methodology to obtain the preferences of a regulator involved in a construction project conflict labelled "The New Recife Project" and subsequently carry out a GMCR study to determine the possible compromise resolutions.

Langenegger and Hipel (2019) use GMCR for strategically investigating the negotiation that took place between the USA and Iran to arrive at an agreement over Iran's nuclear program. In fact, Iran continually escalated the conflict by building more centrifuges needed to produce fissionable nuclear material while the USA responded by stiffening economic sanctions against Iran until both sides finally agreed to negotiate a settlement. The authors also study the role attitudes played in this widely known dispute.

The contributions contained in the four papers mainly dealing with group decision making are now put into perspective. Zhou et al. (2019) examine strategic cooperation from the viewpoint of a weak chain member based on a multi-stage game and bargaining game. They develop game models under different scenarios to investigate the optimal decisions of all stakeholders, and study how to realize strategic cooperation between the weak main manufacturer and the strong supplier. They further design a revenue-sharing contract based on a relationship-specific investment to cause the cooperation to be more effective. A numerical study is discussed based on the case of KLX Inc. and one major manufacturer of Chinese large passenger aircraft to illustrate the superiority of the proposed method.

Considering terrorists' psychological factors in the decision-making process, Ge et 
al. (2019) propose a defensive resource allocation model within the framework of cumulative prospect theory, in which interval values are utilized for representing the uncertainty of reference points and criteria values. Furthermore, a numerical example is utilized to demonstrate how the proposed model can be employed for effectively determining optimal solutions of the defender in counterterrorism resource allocation. In the future, the resource allocation model developed in this paper could be extended by taking into account preference uncertainty, dynamic resource distribution strategy, and other situations.

Zhan et al. (2019) propose a social network uncertain bounded confidence (SNUBC) model for investigating the uncertain opinion evolution having bounded confidence effects, in which opinions of agents involved in a social network could be certain or uncertain. To explore the impact of uncertain opinions and uncertain tolerances on the evolution results in a SNUBC model, theoretical analyses and numerical examples are presented from two aspects: the ratios of agents expressing uncertain opinions and the average widths of uncertain opinions. Afterwards, the authors study the impacts of social networks, uncertain opinions and bounded confidences on the uncertain opinion evolution in the SNUBC model, in which ER random graphs, WS small world networks, and BA scale free networks are utilized to simulate real social networks.

Finally, Wang and Gong (2019) present a chance constraint and uncertainty theory based optimal priority model for group intuitionistic fuzzy preference relations having a normal uncertainty distribution. The results indicate that, while belief degrees in the chance constraints vary, the priorities for alternatives derived from Model (11) and Model (13) always have an inverse relationship, and the priority of four government departments remain the same. Based on expert elicita- tion, this method can be further applied to the multi-attribute issue evaluation being considered such as sustainability index construction.

\section{Acknowledgments}

The Guest Editors would like to express their deep gratitude to the authors for writing such excellent papers. They would also like to thank the many anonymous referees for carefully reviewing the manuscripts and for providing many helpful suggestions for improving them. Additionally, the Guest Editors would like to state their appreciation to the many people who assisted in making GND 2018 a highly successful event which resulted in the dissemination of a rich range of research ideas. Finally, the Guest Editors would like to sincerely thank Mr. Shinan Zhao and Mr. Shuding Chen for their valuable assistance with respect to this JSSSE project.

\section{References}

Chen Y, Kersten G, Vetschera R, Xu H (Editors) (2018). Group decision and negotiation in an uncertain world.18th International Conference, GDN 2018, Nanjing, China June 9-13, 2018, Proceedings, DOI: 10.1007/9783-319-92874-6, Lecture Notes in Business Information Processing 315(LNBIP 315) Springer, Cham Switzerland.

Ge B, Zhang X, Zhou X, Tan Y (2019). A cumulative prospect theory based counterterrorism resource allocation method under interval values. Journal of Systems Science and Systems Engineering 28(4): 478-493.

Jiang X, Xu H, He S, Ke G Y (Editors) (2018). 2018 Proceedings of the 18th International Conference on Group Decision and Negotiation, Nanjing, China June 9-13, 2018, extended abstract, published by the College of Economics and Management, Nanjing University of Aeronautics and Astronautics, Nanjing, China, 2018.

Langenegger T W, Hipel K W (2019). The strategy of escalation and negotiation: The Iran nuclear dispute. Journal of Systems Science and Systems Engineering 28(4): 434-448.

Silva M M, Hipel K W, Kilgour D M, Costa A P C S (2019). Strategic analysis of a regulatory conflict using Dempster-Shafer theory and AHP for preference elicitation. Journal of Systems Science and Systems Engineering 28(4): 415-433.

Wang L, Gong Z (2019). On optimal priority modelling of group intuitionistic fuzzy preference relations with nor- 
mal uncertainty distribution. Journal of Systems Science and Systems Engineering 28(4): 510-525.

Wu Z, Xu H, Ke G Y (2019). The inverse problem with third-party mediation in the graph model for conflict resolution. Journal of Systems Science and Systems Engineering 28(4): 399-414.

Zhan M, Liang H, Ding Z, Dong Y (2019). Uncertain opinion evolution with bounded confidence effects in social networks. Journal of Systems Science and Systems Engineering 28(4): 494-509.

Zhou J, Zhu J, Wang H (2019). Strategic cooperation with differential suppliers' ability under downstream competition in complex product systems. Journal of Systems Science and Systems Engineering 28(4): 449-477.

Keith W. Hipel is university professor of systems design engineering at the University of Waterloo, Officer of the Order of Canada, former President of the Academy of Science (Royal Society of Canada), Senior Fellow of the Centre for International Governance Innovation, Fellow of the Balsillie School of International Affairs, and Coordinator of the Conflict Analysis Group at Waterloo. He received his
B.Sc., M.Sc and Ph.D. degrees from the University of Waterloo in 1970, 1972 and 1975, respectively. He is globally renowned for his interdisciplinary research in systems engineering on the development of conflict resolution, multiple objective decision-making, and time series analysis for addressing complex system of systems problems lying at the confluence of society, technology, and the environment, with applications in water resources, environmental engineering, energy, and sustainable development.

Haiyan $\mathrm{Xu}$ is professor with the College of Economics and Management, Nanjing University of Aeronautics and Astronautics, China. She received her B.Sc. degree in the Department of Mathematics from Nanjing University, China, in 1984, and her M.Sc and Ph.D. degrees in the Department of Combinatorics and Optimization and the Department of Systems Design Engineering from the University of Waterloo in Canada in 2006 and 2009, respectively. Her current research interests in game theory and optimization include the development of normal algebraic techniques for conflict resolution and methods for optimization with applications in environmental management and finance. 